Meta

Journal des traducteurs

Translators' Journal

\title{
Proust, traducteur de Ruskin. De la traduction de Ruskin à la création d'À la recherche du temps perdu
}

\section{Younès Ez-Zouaine}

Volume 62, numéro 3, décembre 2017

La traduction littéraire comme création

URI : https://id.erudit.org/iderudit/1043950ar

DOI : https://doi.org/10.7202/1043950ar

Aller au sommaire du numéro

Éditeur(s)

Les Presses de l’Université de Montréal

ISSN

0026-0452 (imprimé)

1492-1421 (numérique)

Découvrir la revue

Citer cet article

Ez-Zouaine, Y. (2017). Proust, traducteur de Ruskin. De la traduction de Ruskin à la création d’A la recherche du temps perdu. Meta, 62(3), 585-598.

https://doi.org/10.7202/1043950ar
Résumé de l'article

La rédaction de Contre Sainte-Beuve et d'À la recherche du temps perdu de Marcel Proust a été précédée par la traduction de The Bible of Amiens (1884) et de Sesame and Lilies (1865) de John Ruskin. L'examen de ces deux traductions montre que le traducteur s'en est inspiré pour échafauder le socle esthétique de son oeuvre à venir. En effet, la traduction des essais ruskiniens fait partie intégrante du processus d'apprentissage et de maturation qui devance et prépare la rédaction définitive de $\grave{A}$ la recherche du temps perdu. Cette traduction a consisté en une activité de transfert des contenus esthétiques de l'oeuvre de l'esthète anglais à la sienne propre. Nous essaierons dans cet article de montrer que le cadre esthétique d'À la recherche du temps perdu provient directement, avec quelques modifications que nécessite toute transformation créatrice, de sa confrontation avec les livres de Ruskin. 


\title{
Proust, traducteur de Ruskin De la traduction de Ruskin à la création d'À la recherche du temps perdu
}

\author{
YOUNÈS EZ-ZOUAINE \\ Université Mohammed Ben Abdellah, Fès, Maroc* \\ younes.ezzouaine@usmba.ac.ma
}

\section{RÉSUMÉ}

La rédaction de Contre Sainte-Beuve et d'À la recherche du temps perdu de Marcel Proust a été précédée par la traduction de The Bible of Amiens (1884) et de Sesame and Lilies (1865) de John Ruskin. L'examen de ces deux traductions montre que le traducteur s'en est inspiré pour échafauder le socle esthétique de son œuvre à venir. En effet, la traduction des essais ruskiniens fait partie intégrante du processus d'apprentissage et de maturation qui devance et prépare la rédaction définitive de $A$ la recherche du temps perdu. Cette traduction a consisté en une activité de transfert des contenus esthétiques de l'œuvre de l'esthète anglais à la sienne propre. Nous essaierons dans cet article de montrer que le cadre esthétique d'À la recherche du temps perdu provient directement, avec quelques modifications que nécessite toute transformation créatrice, de sa confrontation avec les livres de Ruskin.

\section{ABSTRACT}

The writing of Contre Sainte-Beuve and $\grave{A}$ la recherche du temps perdu was preceded by Marcel Proust's translation of John Ruskin's The Bible of Amiens (1884) and Sesame and Lilies (1865). After a close reading of these two translations, we conclude that Ruskin's texts inspired Proust to construct the theory and aesthetic foundation of his future work. Indeed, the translation of Ruskin's essays is part of the process of maturation that heralds À la recherche du temps perdu. This psychological process seems to occur as Proust aesthetically transfers content from the work of the English aesthete to his own. In this article, we endeavor to demonstrate that the theoretical framework of $\grave{A}$ la recherche $d u$ temps perdu is the direct result of Proust's confrontation with these two works by Ruskin, although it is apparent that the creative process required certain modifications.

\section{RESUMEN}

La traducción por Marcel Proust de The Bible of Amiens (1884) y Sesame and Lilies (1865) de John Ruskin es anterior a la redacción de Contre Sainte-Beuve y À la recherche du temps perdu. El estudio de ambas traducciones muestra que el traductor se inspiró en ellas para sentar los cimientos de su obra futura. Así, forman parte integrante del proceso de aprendizaje y maduración que prepara y conduce a la escritura definitiva de À la recherche du temps perdu. La traducción de esos ensayos consistió en una actividad de transferencia de los contenidos estéticos de la obra del crítico inglés a la suya propia. En este artículo se intentará mostrar que el marco estético de À la recherche du temps perdu proviene directamente de su confrontación con los libros de Ruskin, con algunas modificaciones como lo requiere habitualmente cualquier transformación creativa.

\section{MOTS CLÉS/KEYWORDS/PALABRAS CLAVE}

Proust, Ruskin, transfert esthétique, esthétème, esthétique

Proust, Ruskin, aesthetic transfer, esthetem, aesthetics

Proust, Ruskin, transferencia estética, estetema, estética 
Il n'y a pas de meilleure manière d'arriver à prendre conscience de ce qu'on sent soi-même que d'essayer de recréer en soi ce qu'a senti un maître. (Ruskin 1884/1904: 93) ${ }^{1}$

\section{Introduction}

Pour les éditeurs et traducteurs, la traduction consiste à rendre accessible aux lecteurs d'une langue dite d'arrivée un contenu qui ne l'est que dans la langue dite de départ. Cependant, pour certains écrivains qui s'adonnent à la traduction, celle-ci est plutôt motivée par ce que les philosophes appellent, dans un contexte fort différent du nôtre, sa raison séminale ${ }^{2}$. La traduction d'un écrivain inutile, à première vue, à sa langue, est, généralement, profitable à son œuvre. C'est ce que semble prouver la traduction des deux livres de John Ruskin ${ }^{3}$ par Marcel Proust, La Bible d'Amiens (1884/1904) et Sésame et les lys $(1865 / 1906)^{4}$. Si la traduction proustienne des essais ruskiniens a été, selon nous, sans impact sur le débat intellectuel qui a suivi la mort de l'esthète anglais en 1900, les différentes éditions de ces deux traductions au cours du $\mathrm{xx}^{\mathrm{e}}$ siècle bénéficient depuis la fin des années 1960 de l'éclat ultérieur propre à la personnalité littéraire de Proust ${ }^{5}$ et ne pourraient présenter, à vrai dire, qu'un intérêt anecdotique, pour emprunter le jargon d'une certaine critique contre-sainte-beuvienne.

Comme c'est le cas pour la plupart des écrivains-traducteurs, le transfert dans la traduction des textes de Ruskin ne porte pas seulement sur les contenus sémantiques (comme cela pourrait l'être dans celui, par exemple, de la traduction d'un texte d'idées ou d'un roman d'espionnage), mais aussi sur des contenus esthétiques et théoriques. De ce fait, la traduction de Proust devrait être considérée comme participant d'une activité qui vise à importer de l'œuvre de l'esthète anglais sa thématisation de l'expérience esthétique et quelques-unes des techniques qui lui ont permis de communiquer sa vision aux lecteurs ${ }^{6}$. Cette question de la traduction-transfert nous amène à formuler autrement les termes de notre propos. Au lieu d'aborder cette traduction en partant de la problématique de l'in/fidélité en allant du texte source au texte cible, nous inverserons le processus en remontant du texte de Proust vers celui de Ruskin afin de montrer de quelle manière la traduction des deux livres en question constitue une sorte de sésame à la création d'À la recherche du temps perdu (dorénavant La recherche) ${ }^{7}$.

Si notre interrogation porte essentiellement sur l'activité traductive de Proust, elle ne saurait donc s'abstenir de toucher à l'ensemble de son expérience scripturale. La traduction des essais de Ruskin s'apparente dans le contexte de la création de $\mathrm{La}$ recherche à une matrice qui, si elle n'a pas généré directement l'esthétique de Proust, a contribué à sa cristallisation et, partant, à la naissance de son œuvre. La volonté de les traduire étant motivée par des enjeux à la fois théoriques et techniques, Proust semble y avoir décelé un ressort de la création de son œuvre à venir en opérant un transfert des données esthétiques de l'œuvre ruskinienne à la sienne propre. C'est dans ce sens que nous essaierons de montrer, en recourant à deux métaphores puisées dans La recherche, que Proust a utilisé les essais de Ruskin comme des "verres grossissants» (Le Temps retrouvé, Proust 1927: 338) en vue de traduire "son livre intérieur» (Proust 1927: 186) $)^{8}$. 


\section{La traduction comme initiation}

La traduction s'est présentée à Proust à un moment décisif de sa formation de romancier. À l'encontre de la vocation du protagoniste, Marcel, dans La recherche, celle de l'auteur a été marquée beaucoup plus par la traduction des essais de Ruskin que par une expérience intime de quelque importance qu'elle pût être. Nous émettons donc à l'origine de notre réflexion l'hypothèse selon laquelle le soubassement théorique de son grand œuvre est l'aboutissement d'un échange profond avec la pensée esthétique du critique d'art anglais. Or, si Proust a atteint sa maturité théorique en empruntant le chemin escarpé de la traduction, La recherche devrait être considérée non comme le récit d'une vocation, mais, bel et bien, comme le résultat d'une interaction qui passe par la traduction.

\subsection{Le lieu de la cristallisation}

Le désir de traduire ces deux essais a été déclenché chez Proust par la vive admiration qu'il a ressentie envers Ruskin à la lecture de quelques bribes traduites de son œuvre (Erman 1994: 128). Si le jeune romancier ne cesse, depuis au moins décembre 1895 (Tadié 1996: 599), d'exprimer son enthousiasme pour cette œuvre, c'est qu'il a perçu à l'égard de son auteur une sorte d'affinité intellectuelle. Ne dit-il pas, sous l'effet de l'enthousiasme et de la ferveur, en se référant à Goethe, dans une note de Sésame et les lys, que «l'œil ne perçoit la lumière que parce qu'il est fait de lumière» (Ruskin 1865/1906: 76, note 1) ? Aussi va-t-il finir par apprendre «par cour» (Tadié 1996: 602) plusieurs de ses livres. Toutefois, quoique les biographes soulignent l'intérêt précoce et spontané de Proust pour l'œuvre ruskinienne, ce sont les essais de ses deux exégètes français, L'Esthétique anglaise de Joseph Milsand (1864) et Ruskin et la religion de la beauté de Robert de La Sizeranne (1897), qui lui ont permis d'accéder d'emblée à l'esthétique et à la pensée ruskiniennes et le conduisent à saisir facilement et immédiatement les enjeux théoriques soulevés par l'œuvre ruskinienne.

Il convient de préciser aussi que le désir de traduire qui s'ensuit est symptomatique d'un penchant inconscient propre au génie littéraire de Proust. S’il a déjà publié Les Plaisirs et les jours (1896) et "travaillé depuis très longtemps " (Painter 1966/2008: 319) à la rédaction de Jean Santeuil, le jeune romancier, qui commence à percer à jour les secrets de l'enseignement ruskinien, n'a pas encore trouvé de réponses satisfaisantes et définitives à ses interrogations théoriques. Or, ne pouvonsnous pas faire l'hypothèse que l'abandon de Jean Santeuil, dont la rédaction coïncide avec les premières lectures des textes ruskiniens, est imputable à cet état de crise théorique ?"

Faisant suite à l'impasse théorique et technique de ce roman autobiographique, la traduction conduit Proust de l'échec du projet initial à la promesse d'une œuvre réussie ${ }^{10}$. Étant donné que cette dernière est le fruit d'affirmations intellectuelles soutenues tout au long de sa carrière d'écrivain, nous allons démontrer qu'elles ont été exclusivement acquises dans son commerce avec l'œuvre de Ruskin. S'apparentant à la critique littéraire et au pastiche qu'il pratique pendant toute la période précédant la rédaction de La recherche, la traduction lui permet de mûrir autrement sa conception de l'écriture et de l'art. Le traducteur de Ruskin ne pense, égoïstement, qu'au profit que cette activité de transfert est susceptible d'apporter à son œuvre. Pour 
preuve, dans les nombreuses notes qui entrelacent les textes de The Bible of Amiens et de Sesame and Lilies, le discours proustien devient, à maintes reprises, autoréférentiel. Le traducteur révèle le processus de cristallisation théorique qui a lieu au moment même où s'opère le transfert. Nombre d'indices figurent encore dans les textes des traductions et exhibent une sorte d'état d'âme du traducteur en train de traduire. La traduction des essais de Ruskin n'est pas pour Proust un simple travail de transfert, mais le lieu d'une «découverte» et cette dernière a l'allure inhabituelle d'«un événement» (Tadié 1971: 407).

Si le ton lyrique qu'il adopte en plusieurs endroits des traductions semble empiéter sur la rigueur exigée par une telle activité, en revanche, le cri archimédien est largement justifié par l'importance des trouvailles. Sous l'effet de l'étonnement, Proust s'extasie (Ruskin 1865/1906: 76, note 1), à l'idée d'exprimer la même réflexion que Ruskin, et ce, dit-il, sans l'avoir découverte dans l'un de ses ouvrages lus précédemment. Fort de cette observation, il cite, fébrilement, un passage de son introduction à The Bible of Amiens en vue de se convaincre lui-même (sans doute plus que le lecteur) qu'il a formulé auparavant la même réflexion sans être informé de celle de Ruskin. En effet, la note semble n'avoir d'autre raison que d'exprimer le sentiment du traducteur au moment de sa découverte. S’appuyant sur le manuscrit de la traduction, Tadié confirme qu'elle «a été rédigée, comme en témoignent le style et le manuscrit, avec la hâte qui est le signe de grandes découvertes» (Tadié 1971: 230).

Cependant, cet état de transe dans lequel est plongé le traducteur de Ruskin ne l'empêche pas d'envisager son activité en termes de profit. Proust déclare qu'«à [s]on amour pour les livres de Ruskin se mêla ainsi à l'origine quelque chose d'intéressé, la joie du bénéfice intellectuel qu'[il] allai[t] en retirer» (Ruskin 1884/1904: 90). Cette métaphore mercantile est corroborée quelques pages plus loin par une autre, d'ordre politique: «Aussi cette servitude volontaire est-elle le commencement de la liberté. Il n'y a pas de meilleure manière d'arriver à prendre conscience de ce qu'on sent soimême que d'essayer de recréer en soi ce qu’a senti un maître» (Ruskin 1884/1904: 93). Cet emprunt à La Boétie met en lumière l'effort intellectuel et psychologique auquel s'est livré le romancier en herbe, en «soumettant son esprit à rendre cette vision» (Tadié 1996: 623), pour élaborer une esthétique capable de donner lieu à une œuvre réussie, quelques années plus tard. C'est que Proust croit qu'«on est poussé par autrui sur ses propres voies» (Ruskin 1884/1904: 127) et c'est dans ce sens que s'explique toute la période de préparation qui précède la rédaction de La recherche.

\subsection{De la nécessité de traduire Ruskin}

La traduction n'a pas seulement été pour Proust une occasion de découvrir par luimême la pensée esthétique de Ruskin, mais elle lui a permis de connaître la langue anglaise. C'est qu'en effet celui qui se présente comme étant le traducteur de The Bible of Amiens ne connaît nullement l'anglais au moment de prendre sa décision. En revanche, même s'il ne reconnaît que par intermittences l'aide de ses amis et de sa mère ${ }^{11}$, cette entreprise qui semble avoir été à l'origine une supercherie a donné lieu, indirectement, à un chef-d'œuvre.

En outre, la volonté qu'il déploie pour apprendre l'anglais et, partant, traduire Ruskin, naît de son envie d'échapper à l'infécondité dans laquelle il se trouve vers la fin des années 1890. Plusieurs lettres de la correspondance du traducteur en 
témoignent. Il décidera de s'engager dans cette aventure en dépit de sa méconnaissance de la langue du texte à traduire:

Proust savait très peu l'anglais: au lycée Condorcet, où il fit ses études, les langues qu'il choisit furent le latin, le grec et l'allemand. Même s'il a pris quelques leçons d'anglais, il ne posséda jamais suffisamment cette langue pour pouvoir la traduire et la lire couramment. (Kolb 1960: 22)

Les remarques de Kolb concordent avec celles de Cynthia Gamble dans Proust as Interpreter of Ruskin. The Seven Lamps of Translation. En effet, à la question «Did Proust learn English? », Gamble répond par la négative: "As regards English classes, there is absolutely no evidence to date that Proust attended English lessons at Condorcet» (Gamble 2002: 19). L'importance de sa traduction nous semble bien résider dans le profit qu'elle a apporté à l'auteur. Les incorrections fâcheuses qui subsistent dans la version finale nous conduisent à nous interroger sur son intérêt immédiat, en particulier pour le lecteur des années 1900. Citons Émile Audra:

Le traducteur ignorait, par exemple, que le mot actually a un sens différent du français actuellement; au lieu de traduire par une expression telle que «à vrai dire», il le rend par le mot «aujourd'hui». Ruskin ayant voulu dire que les stalles du chœur d'Amiens sont solides comme il y a quatre cents ans, le traducteur, prenant l'adjectif anglais sound pour un verbe, le traduit «résonnent». (Audra, cité par Kolb 1960:260)

Ces contresens (Tadié 1996: 731) montrent que Proust a cherché à traduire Ruskin pour y puiser des idées qui lui semblaient de nature à irriguer son œuvre à venir. La première traduction n'a donc eu d'autre utilité pour Proust que de rendre compte du fond de la pensée de Ruskin:

Il exista donc toujours entre Proust et l'œuvre de Ruskin une sorte de barrière linguistique que lui imposa son ignorance de l'anglais. Cette barrière dut le gêner en l'empêchant d'accéder directement aux textes. (Audra, cité par Kolb 1960: 267)

La barrière linguistique ne pouvant être dépassée et vu qu'à cette époque aucune traduction complète d'un livre de Ruskin n'était disponible en français, l'auteur de La recherche fut amené à demander l'aide de ses amis (voir supra et note 9). La traduction devait aider le jeune Proust en rupture d'inspiration à lire Ruskin et à satisfaire sa curiosité. Or, une fois effectuée la traduction de quelques extraits, l'auteur de Contre Sainte-Beuve sent le besoin de l'améliorer et, par conséquent, se trouve en train d'accomplir un travail d'approfondissement et de transfert qu'il n'avait pas anticipé.

\section{La traduction comme heuristique}

Le travail réalisé par Proust n'est pas une traduction en bonne et due forme. Il constitue tout au moins la trace finale du processus de recréation qui s'y est produit. Le paratexte critique qui l'accompagne met en scène son investissement psychique et intellectuel à la fois dans l'effort de traduction et dans le développement de sa propre pensée esthétique. En outre, les notes et les préfaces qui phagocytent la traduction s'apparentent à une transcription de conversation où l'auteur traduit exprime ses jugements esthétiques et où le traducteur rétorque en les évaluant à l'aune de ses convictions et de sa conception de l'art, laquelle se fait jour au fur et à mesure de cet échange. 


\subsection{Proust et Ruskin: le nain "ligot[ant] un géant"}

Le rôle tenu par le futur auteur de La recherche dans la traduction des essais de Ruskin sera complété par ceux de préfacier, de commentateur et d'annotateur, ce qui finira par faire oublier sa méconnaissance de l'anglais. Dans La Bible d'Amiens et Sésame et les lys, sur les couvertures desquels figure à côté du nom de Ruskin la mention «Traduit, présenté et commenté par Marcel Proust», la quantité des préfaces, introductions et notes équivaut approximativement au tiers du texte final. En outre, le jeune traducteur a placé délibérément ses commentaires au début des œuvres traduites. Non seulement il n'hésite pas à rédiger des préfaces qui occupent les emplacements stratégiques de ces dernières, mais il couvre aussi les premières pages du texte ruskinien de notes de bas de page, ne laissant à l'auteur que le temps d'esquisser ses idées, sans les éclaircir ni les développer. La note qui accompagne, par exemple, l'épigraphe de Sésame et les lys occupe toute la première page et s'étend sur les deux suivantes, éclipsant totalement le propos de l'auteur qui, jusqu'à la troisième page de sa première conférence, n'est pas encore autorisé à prendre la parole.

En revanche, la plupart de ces notes présentent des explications où le traducteur prétend rendre accessible au lecteur profane le discours hermétique de l'auteur traduit. Force est de constater que l'intention initiale de ces gloses interminables a été de pallier l'hermétisme et l'inachèvement des développements ruskiniens qu'une traduction non accompagnée de commentaires aurait rendus à coup sûr moins accessibles. Proust-annotateur finit cependant par saturer la parole de l'écrivain traduit. Quittant à tout propos sa peau de traducteur, il emprunte le rôle d'un commentateur ennuyeux qui se serait fait passer aux yeux des lecteurs du tournant du $\mathrm{xx}^{\mathrm{e}}$ siècle pour «un nain» cherchant à «ligoter", selon la métaphore de Henry, "à coups de petites notes pressées en caractères menus au bas des pages [...] un géant» (Henry 1981 : 209).

Pour le lecteur moderne, qui a une idée de l'ampleur du projet littéraire de ce jeune commentateur en difficulté, les notes au bas des pages et les préfaces sont le lieu d'un débat théorique entre le maître et le disciple dont l'objectif a été de conduire le second à mettre en forme les principes esthétiques sur lesquels doit se fonder sa somme romanesque à venir. Du fait même qu'elle n'est pas encore achevée (ou qu'elle ne se présente pas dans son état final, comme c'est le cas dans La recherche ou dans Contre Sainte-Beuve), l'esthétique proustienne ne fait qu'éclore dans les textes des traductions. Les pratiques exégétiques qui accompagnent la traduction permettent à Proust d'opérer un retour aux origines de la pensée ruskinienne et de méditer les processus de sa constitution. Se soumettant à la même dialectique que le travail critique, la traduction de Proust est l'occasion d'une activité plus complexe puisqu'elle confond les processus de la compréhension, de l'interprétation et de la réexpression. Le fait de ré-exprimer la pensée ruskinienne en français requiert donc de Prousttraducteur d'être le plus fidèle possible au rapport originel que l'écrivain traduit a installé avec le monde qu'il a créé. D’où l'importance que Proust-traducteur accorde au discours paratextuel qui accompagne ses deux traductions ${ }^{12}$.

Du reste, pour apporter plus de lumière à la spécificité du débat engagé avec Ruskin, nous allons tenter d'en démêler les différentes intentions. Malgré leur hétérogénéité, les discours paratextuels tenus par Proust peuvent être répartis en trois catégories: explicative, commentative et théorique. D'abord, les notes où Proust cherche explicitement à rendre le discours de Ruskin accessible au lecteur profane et 
mondain des années 1900 ont pour objectif d'éclairer l'inintelligible du discours ruskinien. En empruntant un ton didactique, Proust-annotateur clarifie le propos de l'auteur traduit soit en le paraphrasant soit en faisant appel à des extraits puisés dans les textes de ses exégètes (majoritairement, empruntés, sans le signaler explicitement, à de La Sizeranne et à Milsand). Par exemple, lorsque l'esthète anglais affirme que «all great Art is Praise» (Ruskin 1884: vi) ${ }^{13}$, le traducteur intervient, en faisant appel à la Bible d'Amiens et aux autres œuvres de Ruskin, pour expliciter le sens de cette réflexion hermétique pour le lecteur non avisé. Ensuite, les notes commentatives mettent en scène Proust débattant avec Ruskin et montrant la fausseté ou la relativité de ses vues esthétiques, et ce, dans la perspective de mettre en valeur les siennes propres. Par ailleurs, en portant sur des points de détail, ces notes permettent au commentateur de mettre en avant des intuitions qu'il n'arrive pas à thématiser. Dans la note 1 de la page 180, Proust évoque l'érudition de Ruskin sans aller jusqu'à étudier le statut de celle-ci dans son œuvre ni montrer qu'elle participe de sa vision de l'art et de la critique. En effet, dégager une loi générale sur l'investissement du savoir dans l'œuvre d'art nous aurait davantage rapproché de la démarche proustienne dans $L a$ recherche. Or, au lieu de théoriser l'utilisation consciente par Ruskin des connaissances générales, Proust s'extasie devant sa capacité à faire référence aux différentes «draperies déployées aux fenêtres» (Ruskin 1884/1904: 108) dans les tableaux de Carpaccio, sans aller jusqu'à la rattacher à sa conception générale de la critique d'art. Enfin, les notes à portée théorique pallient le tâtonnement inductif propre aux notes commentatives et présentent un traducteur au fait de la pensée ruskinienne et qui tente d'en démêler les tenants et les aboutissants. Ces notes-là sont, précisément, le lieu de l'éclosion des grandes idées qui structureront, des années plus tard, le socle théorique de La recherche et de Contre Sainte-Beuve. En abordant les principes structurants de la pensée esthétique ruskinienne, Proust-annotateur en déduit les paramètres théoriques et les techniques nécessaires à la production et à la réception des œuvres littéraires et artistiques. Au reste, ces notes lui permettent de négocier (et de s'approprier) l'esthétique de Ruskin dans ses moindres articulations.

Si Proust approfondit la théorie esthétique ruskinienne en assurant son passage à la langue française, ses modes d'appropriation et de transfert restent néanmoins tributaires de la spécificité de l'activité traductive. Proust-traducteur découvre la pensée esthétique de Ruskin sous forme de fragments et d'unités discontinues, ce que nous désignons dans cet article du néologisme esthétème forgé sur "philosophème». Par esthétème, nous entendons une unité discontinue d'un discours esthétique considéré comme un système. L'esthétème se différentie du thème par le fait qu'il n'est pas seulement un contenu mais un principe de construction et d'élaboration d'un univers littéraire ou artistique donné. L'ensemble des esthétèmes auxquels a recours un écrivain ou un artiste pour penser sa production constitue ce qu'on appelle couramment une esthétique.

Pour en donner une idée plus précise et le différencier du thème, voyons, à titre d'exemple, le traitement que consacre Proust à l'idolâtrie qui est, selon lui, «le centre de gravité de l'esthétique ruskinienne» (Ruskin 1884/1904: 58). La recherche présente plusieurs personnages idolâtres et snobs, mais le plus représentatif d'entre eux est Legrandin. Du fait de son omniprésence dans l'œuvre de Proust, ce thème finit par fonctionner tel un principe de construction de l'univers romanesque proustien. Aussi le poids de Charles Swann dans La recherche ainsi que sa trajectoire montrent-ils que 
l'idolâtrie constitue un des articles de foi les plus prégnants dans l'esthétique de Proust. N'aimant Odette de Crécy que parce qu'elle ressemble à un personnage dans un tableau de Giotto, Swann finit par illustrer le mal suprême dont Marcel se dégagera à la fin du roman. Or, selon les convictions intellectuelles et morales de l'auteur, la vraie passion de la beauté ne doit pas être en rapport avec un référent extérieur, mais avec la beauté elle-même. À ce niveau de l'analyse, l'idolâtrie devient l'antipode de la mémoire involontaire, laquelle est attachée dans l'univers proustien à un besoin intérieur, d'où la possibilité, exposée à la fin du roman, de créer une œuvre d'art.

Proust part du thème ruskinien concernant l'idolâtrie pour en dégager une loi esthétique sans s'encombrer outre mesure du cas que représente l'auteur évoqué. Si ce mal réside chez Ruskin dans le fait que «son sentiment religieux a dirigé son sentiment esthétique» (Ruskin 1865/1906: 58), Proust en arrive à une idée plus générale: est idolâtre toute personne qui, au lieu de recueillir ses sentiments de la réalité, se laisse influencer par une référence culturelle. Ayant atteint un degré de généralité tel, le thème devient d'ores et déjà un principe théorique sur lequel l'auteur pourrait baser «ses croyances intellectuelles». Mais selon quelles modalités Proust transfère$\mathrm{t}$-il les esthétèmes qu'il a trouvés dans les essais de Ruskin et quelle place leur donnet-il dans son œuvre à venir?

\subsection{La désintégration du texte de Ruskin}

L'appareil critique qui accompagne les textes traduits par Proust montre que le processus traductif revêt l'aspect d'une recréation qui a pour visée de désintégrer le texte même de Ruskin, non en vue de le rendre plus intelligible, mais pour faciliter le transfert de ses unités de base vers un cadre théorique qui s'élabore parallèlement. En prenant ensemble le texte et son commentaire, nous nous rendons compte que le traducteur «fait tout pour n'y mettre que du sien, pour y introduire sa pensée, jusqu'à occulter celle de Ruskin» (Robitaille 2003: 90). La pensée du second devenant presque impossible à distinguer de celle du premier, un mouvement de va-et-vient s'installe entre elles qui empêche de distinguer entre leurs propositions respectives.

Pour s'approprier les esthétèmes ruskiniens, Proust commence par s'emparer de son vocabulaire esthétique. Nous constatons que la terminologie proustienne se forge au contact de celle de Ruskin et à ses dépens. Ne cherchant pas à créer de toutes pièces d'autres concepts, Proust se contente d'emprunter ceux de Ruskin et de les charger d'une signification théorique inédite. L'exemple le plus prégnant est celui de la forme telle que l'entend Ruskin lorsqu'il évoque l'architecture gothique et qu'il interprète en fonction de ses convictions religieuses, alors que Proust l'infléchit dans le sens d'une théorie de l'art et de la littérature (en imitant en cela La Sizeranne) : «and the architectural form can never be well delighted in, unless in some sympathy with the spiritual imagination out of which it rose $»^{14}$, dit Ruskin (1884: 169), en parlant de la chapelle de la cathédrale d'Amiens. Or, en lui opposant une idée de Léon Brunschwig (1900: 97, cité par Proust dans La Bible d'Amiens, Ruskin 1884/1904: 254) qui semble exprimer sa pensée: «Pour éprouver la joie esthétique d'un édifice [...] il faut le sentir en harmonie, non plus avec une quelconque fin extérieure, mais avec l'état intime de la conscience actuelle» (Ruskin 1884/1904: 254), Proust débouche sur une conception opposée à celle de Ruskin, laquelle est détournée définitivement lorsqu’elle figure, inversée, dans une autre formule théorique: «La configuration d'une chose n'est pas 
seulement l'image de sa nature, c'est le mot de sa destinée et le tracé de son histoire» (Ruskin 1884/1904: 57). L'esthétème proustien surgit de l'affirmation ruskinienne. La même rhétorique préside à l'ensemble du traitement proustien du texte qu'il traduit. De ce fait, le lecteur des traductions dispose de deux strates de textes: celle que le traducteur transfère à la langue française, mais aussi celle qu'il lui superpose. Les propositions ruskiniennes n'allant jamais sans écho, le discours de Proust-traducteur semble dédoublé à tel point que nous ne pouvons séparer le texte de la traduction proprement dit de son exégèse.

La deuxième démarche proustienne resitue l'esthétique de l'auteur traduit dans son contexte lointain en révélant au lecteur profane ses ancrages philosophiques. Cette étape de la déconstruction et de la désintégration est basée sur les synthèses de Milsand et de La Sizeranne. Étant ancré dans la même généalogie philosophique (Henry 1981; Fraisse 2013), le traducteur arrive sans trop d'effort à reformuler les esthétèmes ruskiniens en vue de se les approprier. Pire encore, l'auteur de La Bible d'Amiens est décrié au nom des mêmes idées philosophiques qu'il n'arrive pas, selon l'argumentaire proustien, à adapter à la réalité de l'art. La constitution d'un socle esthétique sur lequel se bâtit l'œuvre à venir coïncide avec la recréation de l'œuvre ruskinienne et le transfert de ses unités de base. De ces commentaires sur les idées de Ruskin naît un nouveau dispositif théorique qui, par la voie de la fictionnalisation, donne naissance à plusieurs scènes qui seront reprises dans Contre Sainte-Beuve et La recherche.

\section{La traduction comme transfert esthétique}

Le fait de réduire l'esthétique ruskinienne à ses unités théoriques de base permet à Proust-traducteur de les interroger tout en les préparant à être transférées vers son œuvre à venir. L'auteur de La recherche procède selon deux modalités: l'inversion ou la conversion.

\subsection{La modalité de l'inversion}

Si Proust-traducteur cherche à donner une forme nouvelle au texte source, son intention n'est rien moins que de le reconfigurer, et ce, en vue de l'intégrer à son propre système. Or, loin d'être explicite, il s'évertue à montrer que l'esthétique ruskinienne n'est pas sans présenter des imperfections théoriques, tout en précisant que ses réflexions «constituent [...] une sorte de critique indirecte de [1] a doctrine» (Ruskin 1865/1906: 7, note 1) de l'auteur de Sesame and Lilies. En passant les idées ruskiniennes au crible de ses convictions intellectuelles, Proust frôle souvent la polémique lorsqu'il prétend modifier ce qui ne se conforme pas à la vérité de l'art. D'où l'étape suivante: "en exposant mes idées, je me trouve involontairement [en train de] les opposer d'avance aux siennes» (Ruskin 1865/1906: 7, note 1).

Cette démarche consiste à reprendre les esthétèmes ruskiniens, à y repérer des imperfections et, enfin, à les opposer à d'autres vues théoriques. C'est dans ce sens que dans les passages à portée théorique, l'assertion modalisée et le jugement tendent à remplacer l'analyse et l'exégèse: "Ruskin commet la même erreur» (Ruskin 1884/1904: 57) / «le raisonnement spécieux» (Ruskin 1865/1906: 70) / «il me semble que si le système de Ruskin pèche par quelque côté, c'est par celui-là» (Ruskin 
1884/1904: 57) / «si Ruskin avait tiré les conséquences d’autres vérités qu’il a énoncées quelques pages plus loin, il est probable qu'il aurait rencontré une conclusion analogue à la mienne» (Ruskin 1865/1906: 29). Tirer les conséquences des vérités énoncées? Proust ne s'attribue-t-il pas ce rôle à la place de Ruskin? En effet, le système ruskinien ne semble pas cohérent et c'est à lui, traducteur, d'en réparer les défaillances. Cette audace n'est pas sans rappeler celle de Marx qui affirmait avoir remis la dialectique hégélienne «sur ses pieds». Conformément à la logique qu'il a mise en place, Proust-traducteur n'a plus qu'à inverser les esthétèmes ruskiniens pour obtenir un système esthétique homogène. C'est bien l'inversion des propositions ruskiniennes qui déclenche le processus de création des idées esthétiques proustiennes.

Nous constatons alors que les esthétèmes qui forment la clé de voûte de l'esthétique proustienne dans La recherche sont déduits de ceux formés par Ruskin dans ses deux essais. Référons-nous, par exemple, à l'esthétème de la lecture et au traitement qu'en fait Proust après Ruskin. Si l'esthète anglais croit que la lecture consiste à nous mettre en contact direct avec les esprits intelligents et que nous en apprenons les vérités de l'existence, voici comment Proust inverse la réflexion de l'esthète anglais :

Mais ici, comme du reste très souvent chez les Grecs qui ont dit toutes les choses vraies, mais n'ont pas cherché les vrais chemins plus cachés qui y mènent, la comparaison n'est pas probante. [...] or, même pour ces êtres privilégiés, les amis qu'ils pourront choisir comme ils le voudront ne sauront en aucune façon tenir lieu des livres (ce qui prouve bien que les livres ne sont pas seulement des amis qu'on peut choisir aussi sages que l'on veut parce qu'en réalité ce qui diffère essentiellement entre un livre et une personne ce n'est pas la plus ou moins grande sagesse qu'il y a dans l'une ou l'autre, mais la manière dont nous communiquons avec eux. (Ruskin 1865/1906: 62)

Ni les livres ni leurs auteurs ne nous permettent de saisir la vérité du monde. Dans À l'ombre des jeunes filles en fleur, Proust déclare que personne n'est dispensé de «faire le talent soi-même, par le dedans»; or, comme «il est impossible de faire une bonne santé [bien qu'on manque] à toutes règles de l'hygiène et qu'on commette les pires excès, rien qu'en dînant souvent en ville avec un médecin " (Proust 1919: 150) ${ }^{15}$, on ne peut acquérir une vision juste en fréquentant un écrivain ni en lisant ses livres: «La puissance de notre sensibilité et de notre intelligence, nous ne pouvons la développer qu'en nous-mêmes, dans les profondeurs de notre vie spirituelle» (Ruskin 1865/1906: 50). Si pour Ruskin les livres constituent «le seuil de la félicité spirituelle», pour Proust, en revanche, ils ne sont qu'«une incitation à la réflexion» (Ruskin 1865/1906: 32).

Cette gymnastique imaginative qui consiste à partir des propositions ruskiniennes pour aller vers des affirmations personnelles est inscrite au cœur de la figuration romanesque de La recherche ${ }^{16}$. L'histoire de la vocation de Marcel est opposée à l'infécondité de l'idolâtre, de «l'intelligent Anglais voyageur» (Ruskin 1865/1906: 105) ou du «célibataire de l'art» (Proust 1927: 198). De Swann à Marcel, ne pouvonsnous pas, en effet, résumer le grand mouvement de la fable proustienne? «Du célibataire de l'art» (affilié qu'il est à l'esthétique ruskinienne mais empêtré dans ses signes et sa métaphysique) à "l'artiste» inspiré qu'est Marcel, ne faut-il pas reconnaître le schéma de la progression intellectuelle de La recherche? En ce sens, Proust ne serait pas «contre Ruskin» (Henry 1981: 166), comme il l'est à l'égard de SainteBeuve, mais serait, bel et bien, sa figure inversée. 


\subsection{La modalité de la conversion}

Cependant, la démarche proustienne ne consiste pas toujours à aller contre les propositions ruskiniennes. En d'autres endroits de ses traductions, il se contente de reformuler les esthétèmes ruskiniens en vue de les adapter à son système esthétique. Dans les notes 1 de La Bible d'Amiens (Ruskin 1884/1904:176) et 1 de Sésame et les lys (Ruskin 1865/1906: 61), l'auteur de La recherche reprend la réflexion ruskinienne et cherche à en fortifier soit les principes soit les effets. Par exemple, lorsque Ruskin expose la loi du perspectivisme qui caractérise l'œuvre picturale de Turner, Proust n'hésite pas à la reprendre à son compte et à en défendre le fondement. "Dessiner ce que je vois, non ce que je sais » (Ruskin 1884/1904:66-67) constituera non seulement l'article de foi le plus important de l'esthétique d'Elstir, mais aussi l'un des principes structurants de La recherche.

En outre, la réflexion sur la composition (Ruskin 1865/1906: 177) a été déclenchée par l'usage que Ruskin en fait dans ses conférences ${ }^{17}$. En effet, le travail de fermentation théorique qui commence inopinément lors de la découverte des écrits de Ruskin prend forme avec la trouvaille finale de la structure de La recherche. Même si Proust livre un texte qui semble, au premier abord, labyrinthique, une fois sa lecture achevée, sa structure nous éblouit telle une évidence et nous emplit de l'éclat de sa vérité. La structure qui émerge, selon le canevas même de La recherche, du vécu de l'auteur et de ses échecs, est présentée comme la récompense pour un long travail de recherche et de réflexion. Le passage de Jean Santeuil à La recherche est celui qui se fait de la forme du reportage «chronologique» et de l'exposé linéaire à l'invention d'une écriture achronique et digressive. Les expériences et les découvertes s'y côtoyant, le tout mime le mouvement même de la vie ainsi que les sensations esthétiques qui se dégagent de ces situations réelles. Comme la thématisation de l'expérience esthétique, l'idée de la structure circulaire qui donne forme à La recherche a été développée dans le débat qu'a engagé Proust avec la pensée de l'esthète anglais. Les textes des traductions montrent qu'il n'a pas été indifférent aux techniques de composition adoptées par Ruskin:

Cette épigraphe, [...] projette comme un rayon supplémentaire qui ne vient toucher que la dernière phrase de la conférence (voir page 25), mais illumine rétrospectivement tout ce qui a précédé. (Ruskin 1865/1906: 61)

Le fait de donner à son écriture un tour un peu hermétique en ne révélant les vérités qu'à la fin de son texte est une technique proprement ruskinienne. Proust ne tarde pas à expliciter la technique de composition de Ruskin dans une note de bas de page, dans Sésame et les lys:

Ruskin range l'une à côté de l'autre, mêle, fait manœuvrer et resplendir ensemble toutes les principales idées - ou images - qui ont apparu avec quelque désordre au long de sa conférence. C'est son procédé. Il passe d'une idée à une autre sans aucun ordre apparent. Mais en réalité la fantaisie qui le mène suit ses affinités profondes qui lui imposent malgré lui une logique supérieure. (Ruskin 1865/1906: 69)

Une fois la structure d'ensemble découverte et objectivée, l'auteur n'a plus qu'à dramatiser les motifs ruskiniens et à les intégrer à un schéma existentiel (pour ne pas dire biographique) en vue de donner un sens théorique (donc dogmatique) à une expérience racontée à la première personne du singulier. 
La doctrine esthétique qui se forme au contact de la pensée de Ruskin emprunte à cette dernière jusqu'aux démarches argumentatives qui servent à prouver le bienfondé de ses articles de foi. En offrant à son univers romanesque un arrière-plan démonstratif, Proust invente des scènes romanesques en vue de développer ses vues théoriques. La démarche déductive le conduit de la thématisation de la vision (chose qui se fait dans les annotations qu'il consacre à la traduction de Ruskin et dans les pages théoriques de Contre Sainte-Beuve, mais aussi dans la dernière partie de $\mathrm{La}$ recherche, Le Temps retrouvé) à la trouvaille de la technique (démarche propre aussi à Ruskin) et, enfin, à la création d'épisodes romanesques pouvant démontrer le bienfondé des idées esthétiques qu'il a découvertes (et développées) dans ses discussions avec Ruskin à l'occasion de sa traduction.

\section{Conclusion}

Rappelons que notre hypothèse a consisté à montrer que la traduction des essais de Ruskin a davantage constitué, pour Proust, une étape préparatoire au développement de l'esthétique de son œuvre à venir. C'est dans ce sens que les commentaires et les notes qui accompagnent les textes des traductions ont été considérés comme le lieu d'un débat théorique entre le traducteur et l'auteur traduit. Les modalités de transfert à l'œuvre dans la traduction de The Bible of Amiens et de Sesame and Lilies ont inspiré non seulement la plupart des idées esthétiques proustiennes, mais aussi les scènes romanesques les plus prégnantes dans La recherche.

Par ailleurs, l'étude du transfert esthétique qui a lieu au cours de la traduction proustienne des essais ruskiniens nous a conduit à soulever, sans l'aborder toutefois de façon frontale, la question de la stature et du statut respectifs de l'auteur et du traducteur en général. L'étude du processus de traduction à travers les traces qui nous en restent, à savoir les notes et les commentaires qui accompagnent les versions finales, nous permet de nous interroger sur les limites poreuses qui séparent l'acte d'écrire et celui de traduire. Les fonctions auctoriale et traductoriale nous ont semblé tributaires l'une de l'autre. Nous sommes enfin tenté de dire, plagiant l'auteur du Temps retrouvé, que chaque traducteur est, quand il traduit, le propre traducteur de lui-même.

\section{NOTES}

* Laboratoire «Lettres, Langues et Communication».

1. Ruskin, John (1884/1904): La Bible d’Amiens. (Traduit par Marcel Proust) Paris: Mercure de France.

2. La raison séminale chez les Stoïciens, surtout Plotin (Bréhier 1998), est le "principe actif qui est la loi de l'être et qui, ne faisant qu'un avec la matière, en est comme le germe, en explique le premier mouvement comme les formes» (Dictionnaire en ligne CNRTL). Nous y faisons référence parce qu'elle véhicule une métaphore qui sous-tend notre réflexion sur la question du transfert esthétique. Il s'agit donc d'envisager la traduction des écrivains comme « un intermédiaire entre l'âme et l'être vivant» (Bréhier 1998: 53), autrement dit, entre l'œuvre traduite et celle de l'écrivain-traducteur.

3. John Ruskin (1819-1900) est un écrivain, poète, peintre et théoricien de l'art britannique, dont l'œuvre a été très en vogue tout au long de la deuxième moitié du Xıx ${ }^{\mathrm{e}}$ siècle. Sa doctrine de l'art s'inspire de la théorie du romantisme allemand (surtout Schelling) et en constitue l'accomplissement. Outre ses écrits théoriques, sa fortune et son génie ont été mis au service des peintres préraphaélites et du peintre britannique William Turner dont il a contribué à faire la renommée.

4. Ruskin, John (1865/1906): Sésame et les lys. (Traduit par Marcel Proust) Paris: Mercure de France.

5. La dernière en date est celle établie par Jérôme Bastianelli aux éditions Robert Laffont (collection 
Bouquins) en 2014. Dans la préface qu'Antoine Compagnon consacre à son édition de Sésame et les lys aux Éditions Complexe en 1987, nous lisons à la page 9: "Rééditerait-on aujourd'hui en français Sésame et les lys de Ruskin, si le traducteur n’en avait pas été Proust? Peut-être pas.»

6. Nous opérons d'entrée de jeu une distinction entre les œuvres qui mettent à contribution une esthétique et une théorie de l'écriture, lesquelles font délibérément appel aux procédés littéraires et artistiques, et celles qui se contentent d'informer le lecteur (les textes à facture informative), de le convaincre de la validité d'une thèse (les textes philosophiques) ou de le plonger dans une fiction sans soubassement esthétique (le cas de la paralittérature comme la littérature de gare, les romans policiers, etc.), et ce, même si elles recourent à la forme narrative (ou pseudo-narrative). Par ailleurs, nous entendons par esthétique une conception de l'écriture qui précède la réalisation de l'œuvre d'art et lui donne les caractéristiques de l'homogénéité (une œuvre sans soubassement esthétique regroupe des textes épars) et de l'originalité (une œuvre sans soubassement esthétique ne pourra pas se définir par rapport aux œuvres précédentes ou contemporaines). Le concept de théorie désigne l'ensemble des affirmations intellectuelles qui sont censées répondre à la question de la finalité de l'écriture en la rattachant à l'épistémè en vogue du temps de l'auteur.

7. Pour bien saisir le mouvement qui anime les traductions des écrivains, nous devrions les considérer dans le sens cible source et non l'inverse, contrairement aux études qui les abordent en faisant appel à la problématique de la fidélité/trahison. Ne faut-il pas voir l'intérêt de la traduction par des écrivains dans ce qui la dirige vers un avenir, ce qui lui confère, selon le principe stoïcien de la raison séminale, une fonction de fécondation et de génération d'une autre œuvre?

8. Proust, Marcel (1927/1990): Le Temps retrouvé. Paris: Gallimard.

9. La crise théorique réfère au moment où Proust se trouve incapable de motiver ses choix scripturaux par une conception générale. Aussi l'élan qui, semble-t-il, l'amenait à écrire n'était pas fondé sur des convictions intellectuelles, d'où un sentiment de désespoir et de désillusion envers l'écriture. $\mathrm{Du}$ fait qu'elle est un récit de vocation, La recherche met l'accent sur cet aspect important de la formation du héros, à savoir le moment où Marcel cherche à justifier l'écriture et son importance dans le processus de la quête de la vérité.

10. Tous les spécialistes de Proust conviennent que l'abandon de Jean Santeuil est le résultat d'une immaturité théorique. L'absence de structure et le tâtonnement qui caractérisent sa rédaction montrent assez que son auteur n'avait pas encore trouvé sa vocation, c'est-à-dire son esthétique. Après avoir pris de la distance par rapport à son projet de jeunesse, Proust l'affirme clairement dans une de ses lettres: «Puis-je appeler ce livre un roman? [...] Ce livre n'a jamais été fait, il a été récolté» (cité par Tadié dans sa préface pour Jean Santeuil, p. 7). Voir aussi Henry (1981) qui fait du moment de l'échec de Jean Santeuil le motif de son retour vers les romantiques allemands.

11. Outre l'aide de sa mère, Proust a effectué ses deux traductions grâce à des amis anglais, notamment Marie Nordlinger, Ainslie et Scott, ou des anglicistes comme Robert de Billy et Robert d'Humières (Tadié 1996: 742, 745).

12. Nous nous intéressons aux notes de bas de page plus qu'aux préfaces et aux introductions pour deux raisons. D’un côté, elles ont été écrites simultanément à la traduction proprement dite, contrairement aux préfaces qui l'ont été soit avant, soit après. Aussi la volonté de théoriser y figuret-elle dans l'état de l'hésitation et du tâtonnement. Autrement dit, ces notes se présentent comme étant le journal de bord du traducteur en train d'effectuer sa traduction. D'un autre côté, la note de bas de page fait tellement partie de l'écriture proustienne qu'elle apparaît même dans $L a$ recherche. Tadié écrit: «Cet obscur procédé érudit qu'est la note, si souvent décrié par les écrivains et les critiques, est donc annonciateur de la grande œuvre. Sur les brouillons du roman, les notes sont les additions, les indications de mise en place que Proust se donne à lui-même» (Tadié 1971 : 614, note 2). Notre travail sur la problématique du transfert esthétique au cours de la traduction s'apparente ainsi au questionnement de la critique génétique.

13. Ruskin, John (1884): The Bible of Amiens. Orpington: George Allen.

14. Proust traduit comme suit: «Les formes architecturales ne pourront jamais vraiment nous ravir, si nous ne sommes pas en sympathie avec la conception spirituelle d'où elles sont sorties » (Ruskin 1884/1904: 254).

15. Proust, Marcel (1919/1988): À l'ombre des jeunes filles en fleurs. Paris: Folio classique.

16. De la critique qu'il adresse à Ruskin (et à lui-même, pendant qu'il écrit Jean Santeuil) émergent les premiers épisodes de La recherche: le vice de l'idolâtrie qu'il diagnostique chez l'auteur de The Bible of Amiens fait l'objet des premiers épisodes de La recherche et permet d'exposer un argument qui se trouve être développé dans tout le roman. Swann (qui, le premier, initie Marcel à l'œuvre de Giotto et de Botticelli) et Legrandin sont présentés comme des modèles à éviter pour atteindre la 
vocation artistique. En outre, ces deux personnages sont les équivalents romanesques d'un personnage unique, à savoir l'anti-artiste ruskinien qu'il cherche à déconstruire.

17. Voici ce qu'avance Ruskin: «Toutes les fois que dans un paragraphe ou un chapitre je promets pour le chapitre suivant un examen attentif de quelque point particulier, le paragraphe suivant n'a trait en quoi que ce soit au point promis, mais ne manque pas de s'attacher passionnément à quelque point antithétique, antipathique ou antipodique, dans l'hémisphère opposé; je trouve cette façon de composer un livre extrêmement favorable à l'impartialité et la largeur des vues» (Ruskin 1884/1904: 177).

\section{RÉFÉRENCES}

Brehier, Émile (1998): La philosophie de Plotin. Paris: Vrin.

BRUnSChWig, Léon (1900): Introduction à la vie de l'esprit. Paris: Alcan.

Erman, Michel (1994): Marcel Proust, une biographie. Paris: La petite vermillon.

Fraisse, Luc (2013): L'éclectisme philosophique de Marcel Proust. Paris: Presses de l'Université Paris-Sorbonne.

Gamble, Cynthia (2002): Proust as Interpreter of Ruskin: The Seven Lamps of Translation. Birmingham: Summa Publications.

Henry, Anne (1981): Proust. Théories pour une esthétique. Paris: Klincksieck.

KolB, Philip (1960): Proust et Ruskin: nouvelles perspectives. Cahiers de l'Association internationale des études françaises. 12:259-273.

La Sizeranne, Robert de (1897): Ruskin et la religion de la beauté. Paris: Hachette.

Milsand, Joseph Antoine (1864) : L'esthétique anglaise: étude sur M. John Ruskin. Paris: Libraireéditeur Germer Baillière.

Painter, George (1966/2008): Marcel Proust (1871-1922). (Traduit par Georges Cattaui et R.-P. VIAL) Paris: Texto.

Robitaille, Martin (2003): Proust épistolier. Espace littéraire. Montréal: Les Presses de l'Université de Montréal.

TADIÉ, Jean-Yves (1971): Proust et le roman. Paris: Gallimard.

TAdIÉ, Jean-Yves (1996) : Marcel Proust II. Une biographie. Paris: Gallimard. 